\title{
Identify Certain Physiological Indices Associated with Tillering, Number of Leaves and Leaf Area in Tolerance in Rice
}

\author{
Rita Tiwari ${ }^{1}$, S. P. Singh ${ }^{2}$ \\ ${ }^{1}$ Department of Botany, M.L.K. (P.G.) College Balrampur- 271201 (U.P.) India \\ ${ }^{2}$ Indian Institute of Sugarcane Research, Dilkusha, Lucknow-226 002 (U.P.), India
}

\begin{abstract}
Number of tillers per hill under each treatment was recorded by visual counting of the appropriate stage. The plants already tagged for this purpose were used and average tillers per hill were computed. More numbers of tillers shown in tolerant varieties- Usar Dhan -3 and CSR-23 \& less in susceptible IR-42, CSR-23 total number of leaves were counted for three randomly selected plants at different successive stage of crop growth i.e. 15, 45 and 75 days and expressed as number of leaves hill. More number of leaves shown in tolerant verieties (Usar Dhan-3, CSR-23) in comparison to susceptible varieties (IR-42, CSR-28).
\end{abstract}

Keywords: Rice, salt tolerant, salt sensitive sodic soil, tillering, reproductive stage.

\section{Introduction}

Rice (Oryza sativa L.) is the most important cereal crop that provides $43 \%$ of calories requirement for more than $70 \%$ Indian as well as world population that is why the rice production always holds a key in the over all food situation of the whole world salinity is an important environmental factors that limits growth and field of rice. It currently affects, about 21.5 mha of soil in Asia. (Laffitte et al; 2004) Rice productivity in salt affected area is currently very low 1-15 tha- ${ }^{1}$ but can reasonably be raised by atleast 2 tha- ${ }^{1}-$ (Ponnampruma 1994) by adoption of salt tolerant rice varieties and suitable management practice.

In salt affected soil, rice is generally cultivated by transplanting method where nursery is first grown on normal soil and then it a shifted to the main field at the age of 35 days. Salinity affected rice crop production in many rice growing areas (Funakawa et al, 2000, Kotb et al; 2000, Wison et al; 2000) owing to osmotic stress affecting water absorption, seed germination and seedling growth. There are numerous studies to characterize tolerance at early seedling or vegetative stage but little work has been done under transplanted condition where crop experience salt stress condition from tiller up to reproduction stage only. Looking into the large extent of genotypic variability in rice, the identification of traits for tillering and reproductive stage tolerance can provide and in sight to screening of large number of germplasm along with breeding for genotypes, having salt tolerance during reproduction stage.

\section{Materials and Methods}

The experiment were conducted for two kharif seasons during 2010-11 and 2011-12. The investigation were carried out in earthen pot culture with four varieties/genotypes of rice (Oryza sativa L.) viz. Salt tolerant (Usar Dhan-3 and CSR-23) and salt sensitive (IR-42 and CSR-28) at the experimental site of the departmental of Botany, M.L.K.P.G. college, Balrampur in collaboration with Narendra Dev
University of Agriculture and Technology, crop-physiology department, Kumarganj Faizabad. The experiments were laid out in complete randomized design with four stages and two tips of soil all in three replicates. Number of tillers per hill under each treatment was recorded by visual counting at the appropriate stage. The plants already tagged for this purpose were used and average tillers per hill was counted and the total number of leaves were counted for three randomly selected plants at different successive stages of crop growth i.e. 15,45 and 75 days and expressed as number of leaves per hill. The leaf area of three randomly selected plants were measured with the help of automatic leaf area metre (LICOR-USA model LI-3000) at 15, 45 and 75 DAS after transplanting and then it was average out for one hill.

\section{Result and Discussion}

The number of tillers per hill also an important yield parameters under sodicity because it determines the grain bearing panicles sodicity caused significant reduction in number of tillers will compared is control treatment in this study Under salt stress maximum reduction in tillers was observed IR-42 and CSR-28 at 50 ESP at all growth stages however, minimum was observed in Usar Dhan-3. A highly significant reduction in number of tillers hill ${ }^{-1}$ under sodicity was also reported in rice (Zeng et al ; 2000). The decrease in tillering capacity might be due to the toxic effect of salt on plant growth. The development of more tillers in tolerant varieties may be due to mechanisms of salt tolerance (Aslam et al ; 1989). A significant reduction was observed in leaf number and leaf area due to sodicity in all the rice cultivars, however tolerant verieties, Usar Dhan-, CSR-23 showed comparatively less reduction than IR-42 and CSR-28. The minimum number area of leaves observed at $50 \mathrm{ESP}$ at each growth stages. The result corroborates with Bal and Dutt (1984) other workers have also found reduction in number of leaves and leaf area of different crops under sodicity (Cramer et al; 1994, Batella et al; 1997 and Srivastava, 1998). 


\section{International Journal of Science and Research (IJSR) \\ ISSN (Online): 2319-7064}

Index Copernicus Value (2013): 6.14 | Impact Factor (2015): 6.391

Table 1: No. of Tillers

\begin{tabular}{|c|c|c|c|c|c|c|c|c|c|c|c|c|c|c|c|c|c|c|c|c|}
\hline \multirow[t]{2}{*}{ Varieties } & \multicolumn{5}{|c|}{15 DAYS } & \multicolumn{5}{|c|}{45 DAYS } & \multicolumn{5}{|c|}{75 DAYS } & \multicolumn{5}{|c|}{ AT MATURITY } \\
\hline & SO & $S 1$ & $S 2$ & S3 & Mean & SO & $S 1$ & $S 2$ & S3 & Mean & SO & S1 & $S 2$ & S3 & Mean & SO & $S 1$ & $S 2$ & S3 & Mean \\
\hline $\begin{array}{c}\text { Usar } \\
\text { Dhan-3 }\end{array}$ & 4.35 & 4.19 & 4.07 & 3.98 & 4.15 & 8.24 & 7.95 & 7.69 & 7.54 & 7.86 & 9.92 & 9.55 & 9.26 & 9.06 & 9.45 & 11.19 & 10.75 & 10.45 & 10.24 & 10.66 \\
\hline CSR-23 & 4.14 & 3.98 & 3.86 & 3.79 & 3.94 & 7.78 & 7.49 & 7.24 & 7.11 & 7.41 & 9.50 & 9.15 & 8.86 & 8.69 & 9.05 & 10.92 & 10.50 & 10.20 & 10.00 & 10.41 \\
\hline IR-42 & 3.53 & 3.37 & 3.20 & 3.10 & 3.30 & 7.17 & 6.84 & 6.50 & 6.30 & 6.70 & 8.78 & 8.38 & 7.98 & 7.71 & 8.21 & 9.58 & 9.14 & 8.69 & 8.41 & 8.96 \\
\hline CSR-28 & 3.43 & 3.26 & 3.10 & 3.01 & 3.20 & 7.27 & 6.94 & 6.60 & 6.39 & 6.80 & 8.73 & 8.33 & 7.91 & 7.66 & 8.16 & 9.37 & 8.93 & 8.50 & 8.24 & 8.76 \\
\hline Mean & 3.86 & 3.70 & 3.56 & 3.47 & 3.65 & 7.62 & 7.31 & 7.01 & 6.84 & 7.19 & 9.23 & 8.85 & 8.50 & 8.28 & 8.72 & 10.27 & 9.83 & 9.46 & 9.22 & 9.69 \\
\hline SEm+ & \multicolumn{5}{|c|}{$\mathrm{V}=0.05, \mathrm{~S}=0.05, \mathrm{VXS}=0.10$} & \multicolumn{5}{|c|}{$\mathrm{V}=0.10, \mathrm{~S}=0.10, \mathrm{VXS}=0.20$} & \multicolumn{5}{|c|}{$\mathrm{V}=0.11, \mathrm{~S}=0.11, \mathrm{VXS}=0.23$} & \multicolumn{5}{|c|}{$\mathrm{V}=0.14, \mathrm{~S}=0.14, \mathrm{VXS}=0.28$} \\
\hline $\begin{array}{c}\text { CD at } \\
5 \%\end{array}$ & \multicolumn{5}{|c|}{$\mathrm{V}=0.15, \mathrm{~S}=0.15, \mathrm{VXS}=\mathrm{NS}$} & \multicolumn{5}{|c|}{$\mathrm{V}=0.29, \mathrm{~S}=0.29, \mathrm{VXS}=\mathrm{NS}$} & \multicolumn{5}{|c|}{$\mathrm{V}=0.32, \mathrm{~S}=0.32, \mathrm{VXS}=\mathrm{NS}$} & \multicolumn{5}{|c|}{$\mathrm{V}=0.39, \mathrm{~S}=0.39, \mathrm{VXS}=\mathrm{NS}$} \\
\hline
\end{tabular}

Table 2: No. of Leaf

\begin{tabular}{|c|c|c|c|c|c|c|c|c|c|c|c|c|c|c|c|c|c|c|c|c|}
\hline \multirow[t]{2}{*}{ Varieties } & \multicolumn{5}{|c|}{15 DAYS } & \multicolumn{5}{|c|}{45 DAYS } & \multicolumn{5}{|c|}{75 DAYS } & \multicolumn{5}{|c|}{ AT MATURITY } \\
\hline & S0 & S1 & S2 & S3 & Mean & S0 & S1 & S2 & S3 & Mean & S0 & S1 & S2 & S3 & Mean & S0 & $\mathrm{S} 1$ & S2 & S3 & Mean \\
\hline $\begin{array}{c}\text { Usar } \\
\text { Dhan-3 }\end{array}$ & 13.77 & 13.21 & 12.85 & 12.60 & 13.11 & 46.31 & 44.50 & 43.23 & 42.33 & 44.09 & 50.60 & 48.66 & 47.22 & 46.27 & 48.19 & 43.77 & 42.10 & 40.90 & 40.00 & 41.69 \\
\hline CSR-23 & 10.03 & 9.65 & 9.35 & 9.07 & 9.53 & 44.98 & 43.25 & 41.95 & 41.15 & 42.83 & 53.80 & 51.71 & 50.20 & 49.15 & 51.22 & 46.42 & 44.62 & 43.30 & 42.45 & 44.20 \\
\hline IR-42 & \begin{tabular}{|l|}
9.72 \\
\end{tabular} & 9.30 & 8.80 & 8.56 & 9.10 & 39.60 & 37.75 & 35.90 & 34.77 & 37.01 & 40.25 & 38.35 & 36.50 & 35.35 & 37.61 & 31.00 & 29.48 & 28.05 & 27.20 & 28.93 \\
\hline CSR-28 & 9.05 & 8.65 & 8.20 & 7.95 & 8.46 & 33.00 & 31.44 & 29.89 & 28.98 & 30.83 & 34.76 & 33.15 & 31.52 & 30.55 & 32.50 & 27.30 & 26.00 & 24.75 & 24.00 & 25.51 \\
\hline Mean & 10.64 & 10.20 & 9.80 & 9.55 & 10.05 & 40.97 & 39.24 & 37.74 & 36.81 & 38.69 & 44.85 & 42.97 & 41.36 & 40.33 & 42.38 & 37.12 & 35.55 & 34.25 & 33.41 & 35.08 \\
\hline SEm+ & \multicolumn{5}{|c|}{$\mathrm{V}=0.14, \mathrm{~S}=0.14, \mathrm{VXS}=0.29$} & \multicolumn{5}{|c|}{$\mathrm{V}=0.58, \mathrm{~S}=0.58, \mathrm{VXS}=1.17$} & \multicolumn{5}{|c|}{$\mathrm{V}=0.63, \mathrm{~S}=0.63, \mathrm{VXS}=1.25$} & \multicolumn{5}{|c|}{$\mathrm{V}=0.52, \mathrm{~S}=0.52, \mathrm{VXS}=1.04$} \\
\hline $\begin{array}{c}\mathrm{CD} \text { at } \\
5 \%\end{array}$ & \multicolumn{5}{|c|}{$\mathrm{V}=0.41, \mathrm{~S}=0.41, \mathrm{VXS}=\mathrm{NS}$} & \multicolumn{5}{|c|}{$\mathrm{V}=1.65, \mathrm{~S}=1.65, \mathrm{VXS}=\mathrm{NS}$} & \multicolumn{5}{|c|}{$\mathrm{V}=1.77, \mathrm{~S}=1.77, \mathrm{VXS}=\mathrm{NS}$} & \multicolumn{5}{|c|}{$\mathrm{V}=1.47, \mathrm{~S}=1.47, \mathrm{VXS}=\mathrm{NS}$} \\
\hline
\end{tabular}

Table 3: Leaf area

\begin{tabular}{|c|c|c|c|c|c|c|c|c|c|c|c|c|c|c|c|c|c|c|c|c|}
\hline \multirow[t]{2}{*}{ Varieties } & \multicolumn{5}{|c|}{$15 D A Y S$} & \multicolumn{5}{|c|}{$45 D A Y S$} & \multicolumn{5}{|c|}{$75 D A Y S$} & \multicolumn{5}{|c|}{ AT MATURITY } \\
\hline & SO & S1 & $S 2$ & $S 3$ & Mean & SO & S1 & $S 2$ & $S 3$ & Mean & SO & $S 1$ & $S 2$ & $S 3$ & Mean & SO & S1 & $S 2$ & $S 3$ & Mean \\
\hline & 294 & 283.2 & 274.7 & 269.2 & 280.3 & 438.6 & \begin{tabular}{|l|l}
421.9 \\
\end{tabular} & 409.3 & 401.0 & \begin{tabular}{|l|l|}
417.7 \\
\end{tabular} & 633.9 & \begin{tabular}{|l|l}
6009.8 \\
\end{tabular} & 591.6 & 579.5 & \begin{tabular}{|l|l}
6003.7 \\
\end{tabular} & \begin{tabular}{|l|l|}
607.4 \\
\end{tabular} & 584.4 & 567.0 & 555.5 & 578.5 \\
\hline Dhan-3 & 40 & 0 & 5 & 0 & 9 & 0 & 0 & 0 & 0 & 0 & 0 & 0 & 5 & 0 & 1 & 0 & 0 & 5 & 0 & 9 \\
\hline \multirow[t]{2}{*}{ CSR-23 } & 283 & 272.9 & 264.8 & 259.4 & 270.2 & 25.9 & 409.6 & 397.5 & 389.4 & 405.6 & 640.6 & 615.3 & 597.0 & 584.8 & \begin{tabular}{|l|l|}
609.4 \\
\end{tabular} & \begin{tabular}{|l|l}
617.3 \\
\end{tabular} & 593.7 & \begin{tabular}{|l|}
576.1 \\
\end{tabular} & 564.4 & \begin{tabular}{|l|}
587.9 \\
\end{tabular} \\
\hline & 70 & 0 & 0 & 0 & 0 & 0 & 5 & 0 & 0 & 1 & 5 & 0 & 0 & 0 & 4 & 0 & 5 & 5 & 0 & 0 \\
\hline \multirow[t]{2}{*}{ IR-42 } & 257 & 245.1 & 233.1 & 225.9 & 240.3 & 41.1 & 325.2 & 309.2 & 299.7 & 318.8 & 482.7 & 460.1 & 437.5 & 424.0 & 451.1 & 457.0 & 435.6 & 414.2 & 401.4 & 427.0 \\
\hline & 10 & 0 & 0 & 0 & 0 & 0 & 0 & 0 & 0 & 0 & 0 & 5 & 5 & 5 & 1 & 0 & 5 & 5 & 5 & 9 \\
\hline \multirow[t]{2}{*}{ CSR-28 } & 248 & 237.1 & 225.5 & 218.5 & 232.4 & 326.9 & 311.5 & 296.3 & 287.1 & 305.4 & 477.8 & 455.5 & 433.2 & 419.8 & 446.5 & 448.1 & 427.1 & 406.2 & 393.7 & 4418.7 \\
\hline & 70 & 0 & 0 & 0 & 5 & 0 & 5 & 0 & 5 & 8 & 5 & 0 & 0 & 0 & 9 & 0 & 0 & 5 & 0 & 9 \\
\hline \multirow[t]{2}{*}{ Mean } & 270 & 259.5 & 249.5 & 243.2 & 255.8 & 383.1 & 367.0 & 353.0 & 344.3 & 361.9 & 558.7 & 535.1 & 514.8 & 502.0 & 527.7 & \begin{tabular}{|l|l|}
532.4 \\
\end{tabular} & 510.2 & 4490.9 & \begin{tabular}{|l|l|}
478.7 \\
\end{tabular} & 503.0 \\
\hline & 98 & 8 & 4 & 5 & 3 & 3 & 8 & 8 & 1 & 0 & 8 & 9 & 5 & 4 & 1 & 5 & 3 & 3 & 6 & 9 \\
\hline SEm+ & \multicolumn{5}{|c|}{$\mathrm{V}=3.64, \mathrm{~S}=3.64, \mathrm{VXS}=7.28$} & \multicolumn{5}{|c|}{$\mathrm{V}=0.14, \mathrm{~S}=0.14, \mathrm{VXS}=0.29$} & \multicolumn{5}{|c|}{$\mathrm{V}=7.62, \mathrm{~S}=7.62, \quad \mathrm{VXS}=15.24$} & \multicolumn{5}{|c|}{$\mathrm{V}=7.84, \mathrm{~S}=7.84, \mathrm{VXS}=15.69$} \\
\hline $\begin{array}{c}\text { CD at } \\
5 \%\end{array}$ & \multicolumn{5}{|c|}{$\begin{array}{c}\mathrm{V}=10.29, \mathrm{~S}=10.29, \mathrm{VXS}= \\
\mathrm{NS}\end{array}$} & \multicolumn{5}{|c|}{$\mathrm{V}=10.29, \mathrm{~S}=10.29, \mathrm{VXS}=\mathrm{NS}$} & \multicolumn{5}{|c|}{$\mathrm{V}=21.53, \mathrm{~S}=21.53, \mathrm{VXS}=\mathrm{NS}$} & \multicolumn{5}{|c|}{$\mathrm{V}=22.16, \mathrm{~S}=22.16, \mathrm{VXS}=\mathrm{NS}$} \\
\hline
\end{tabular}

\section{References}

[1] Aslam, M;R.H; Qureshi, N. Ahmed and S.Muhammad (1989). Salinity tolerance in rice (Oryza sativa L.) Morphological studies Pak.J Agric Sci, 26:92-98.

[2] Ashraf ,M (2004)- Some important physiological selection criteria for salt tolerance in plants Vora, 1991361-3/6.

[3] Bernstein, L. (1975)- Effect of salinity and sodicity or plant growth. Ann. Rev. Photopathol 13"295-312.

[4] Goldsworthy, A (1994) calcium and salinity App. Bio; 4 : 1-6.

[5] Funakawa, S. Suzuku , R. Kartoozara E.kosaki, T. and Ishida, N (2000) salt affected 50 is under rice-based irrigation agriculture in southern kazakhastan. Geoderma, 97:61-85.

[6] Lafitte, H.R. 25 mail, A and Benett J (2004). New directions for a diverse plant. Proc $4^{\text {th }}$ International crop
Sci Cong., 26 September 1 October ISBN 1920842209 Brisbane, Australia.

[7] Zing, L and M.C. Shannon (2000). Salinity effects on seedling growth and field components of rice. Crop sci; $40: 998-1003$. 